\title{
ANALYTIC LEFT ALGEBRAIC GROUPS. II ( $\left.{ }^{1}\right)$
}

BY

\author{
ANDY R. MAGID $\left({ }^{2}\right)$
}

\begin{abstract}
An analytic left algebraic group is a complex analytic group carrying a structure of affine algebraic variety such that left translations by fixed elements are morphisms. The core of such a group is the (algebraic) subgroup of all elements such that right translation by them is a morphism. It is shown that the core determines the left algebraic structure, and this is used to determine when left algebraic structures are conjugate by inner automorphisms.
\end{abstract}

A left algebraic structure on a complex analytic group $G$ is a structure of affine algebraic variety on $G$, compatible with the analytic structure on $G$, such that all left translations on $G$ by elements of $G$ are morphisms of the variety structure: that is, there is a finitely generated complex algebra $A$ of holomorphic functions on $G$ such that the maximal ideal space of $A$ coincides, as a set, with $G$, and such that if $f$ is in $A$ and $x$ is in $G$, the function $f \cdot x$ on $G$ given by $(f \cdot x)(y)=f(x y)$ is also in $A$. We refer to the pair $(G, A)$ as an analytic left algebraic group. If a fixed structure is referred to, we denote the pair by $G$ alone and denote $A$ by $C[G]$.

These structures were introduced by Hochschild and Mostow [3, p. 116] in a study of the algebra of analytic representative functions on an analytic group. They showed that every faithfully representable analytic group $G$, which must be a semidirect product of a simply connected solvable normal subgroup and a reductive subgroup, carries at least one left algebraic siructure [3, 3.1, p. 118]. In [6, Definition 1.2], we considered the core $C(G)$ of an analytic left algebraic group $G$ : this is the subgroup $G$ of all elements such that right translation by them is a morphism; equivalently, if $f$ is in $C[G]$ and $x$ is in $C(G)$ the function $x \cdot f$ on $G$ given by $(x \cdot f)(y)=f(y x)$ is in C[G]. It turns out that $C(G)$ is, as a sub left algebraic group of $G$, an algebraic group [6, Theorem 1.7] such that $G \times C(G) \rightarrow G$ by multiplication is a morphism; alternatively, $C(G)$ can be characterized as the maximal subgroup of $G$ with these properties [6, Corollary 1.9].

In this paper, we consider the relations among the cores of various left

Received by the editors August 30, 1976.

AMS (MOS) subject classifications (1970). Primary 20G20; Secondary 22E10.

(') Part I is to appear in the American Journal of Mathematics.

(2) Partially supported by NSF Grant No. MPS73-08482. 
algebraic structures on a fixed analytic group $G$. It is shown that if the cores of two such structures coincide as algebraic groups, then the structures are equal [(1.10) and (2.3)], and if two such structures arise from the same simply connected solvable normal subgroup $K$ of $G$, they are conjugate under inner automorphism by an element of the smallest normal subgroup $N$ of $K$ such that $K / N$ is nilpotent [(1.11) and (2.6)]. (This latter result is a mild generalization of $[3,4.1$, p. 120], where the structures are shown to be conjugate under an element of the radical of the commutator subgroup of $G$.)

The plan of the paper is as follows: in $\$ 1$, we study simply connected solvable analytic left algebraic groups, beginning with a study of the algebraic hull of such a group. The major tool is (1.6), which displays every such group $G$ as a product (not necessarily semidirect) of a Zariski-closed unipotent normal subgroup $N$ and a Zariski-closed unipotent subgroup $C$ such that $N \times C \rightarrow G$ is a morphism of varieties. This allows a calculation of $C(G)$, and it is shown in (1.9) that $\operatorname{Lie}(C(G))$ is a Cartan subalgebra of $\operatorname{Lie}(G)$. The uniqueness and conjugacy results mentioned above now follow from properties of Cartan subalgebras. It should be noted that (1.6) can be established also by analyzing a standard left algebraic structure on $G$ and then applying the conjugacy theorem $[3,4.1, \mathrm{p}$. 120]. This has not been done here for two reasons: first, because we want to derive the conjugacy theorem (1.11) as a consequence, and second, because the proof of $[3,4.1]$ relies on results which give the position of $C[G]$ inside the ring of representative functions on $G$, while the proof of (1.6) can be carried out directly in terms of algebraic and analytic group theoretic properties of the left algebraic group $G$ and its algebraic hull.

In $\$ 2$, we consider analytic left algebraic groups in general, which are semidirect products of simply connected solvable left algebraic groups and reductive, hence algebraic, groups. As noted above, we show in (2.3) that such structures are determined by their cores, where the latter is regarded as an algebraic group. Since a core must contain a maximal reductive subgroup of the entire analytic group, it is the unipotent radical of the core which is the key variable component. We show in (2.5) that in fact a core is the normalizer in the entire group of its unipotent radical. Also, in (2.4) we give necessary and sufficient conditions for a subgroup to be the unipotent radical of the core of some left algebraic structure.

Notations and conventions of [6] are used throughout.

We begin by considering the action of the algebraic hull $G^{\prime}$ of an analytic left algebraic group $G$ on $G$.

1.

Proposition 1.1. Let $G$ be an analytic left algebraic group and let $V$ be $a$ 
finite dimensional subspace of $\mathrm{C}[G]$ which generates $\mathrm{C}[G]$ as a $\mathbf{C}$-algebra and which is a right $G$-submodule of $\mathbf{C}[G]$. Let $W=\operatorname{Hom}(V, \mathbf{C})$, regarded as a left $G$-module, let $p: G \rightarrow G L(W)$ be the associated representation, and let $G^{\prime}$ be the closure of $p(G)$ in $G L(W)$. Then:

(a) There is a vector $w$ in $W$ such that $G w$ is Zariski-closed in $W$ and $G \rightarrow G w$ by $g \rightarrow g w$ is an isomorphism.

(b) $G w$ is $G^{\prime}$-stable.

(c) If $N$ is a subgroup of $G$ such that $p(N)$ is a unipotent algebraic subgroup of $G^{\prime}$, then $N$ is a Zariski-closed algebraic subgroup of $G$ and $N \times G \rightarrow G$ by $(u, g) \rightarrow n g$ is a morphism.

(d) If $G$ is simply connected and solvable as an analytic group and $N$ is an analytic subgroup of $(G, G)$ then $p(N)$ is closed in $G^{\prime}$. Moreover, $(G, G)=\left(G^{\prime}\right.$, $\left.G^{\prime}\right)$.

Proof. (a) Let $S$ be the symmetric algebra of $V$. We have by assumption a surjection $t: S \rightarrow \mathrm{C}[G]$ of $\mathbf{C}$-algebras and right $G$-modules. Then $h$ induces a closed immersion $t^{*}: G \rightarrow W$ of affine varieties, with $t^{*}(g)(f)=f(g)$ for $f$ in $V$. Then $t^{*}(g h)=g \cdot t^{*}(h)$ for $g, h$ in $G$, so if $w=t^{*}(e), t^{*}(g)=g w$. This establishes part (a).

(b) Let $T=\operatorname{Trans}(G w, G w)=\{g \in G L(W) \mid g(G w) \subset G w\}$. Since $G w$ is closed in $W, T$ is a closed subgroup of $G L(W)$ by [1, p. 97]. But $G$ is a subgroup of $T$ and $G^{\prime}$ is the closure of $G$ in $G L(W)$. Thus $G^{\prime}$ is contained in $T$, and part (b) is established.

(c) By assumption, $p(N)$ is unipotent, and $p(N)$ acts on the affine variety $G w$. Since $G^{\prime}$ acts rationally on $G w$ by (b), $p(N)$ acts rationally on $G w$, and it is known [7, Theorem 2, p. 221] that under unipotent actions on affine varieties all orbits are closed. Thus $p(N) w$ is closed in $G w$, and hence $N$ is closed in $G$. As remarked previously $G^{\prime} \times G w \rightarrow G w$ is a morphism, and this implies that $N \times G \rightarrow G$ is a morphism.

(d) Since $G$ is solvable, so is $G^{\prime}\left[1\right.$, p. 109], hence $G^{\prime}=U \cdot T$ where $U$ is unipotent and $T$ is a torus. Then $p((G, G))$ is contained in $U$ and $p: N \rightarrow U$ is an analytic injection between simply connected nilpotent Lie groups. Using canonical coordinates on $U$, it is clear that $p(N)$ is a Zariski-closed subgroup of $U$. Now apply part (c). To complete the proof of (d), we use [1, 2(e), p. 105], to see that $\left(G^{\prime}, G^{\prime}\right)$ is the Zariski closure of $(G, G)$. But this latter subgroup has already been shown to be closed.

We recall [6] that $G^{\prime}$ is in part independent of the choice of $V$; for the coordinate ring of $G^{\prime}$ is just the smallest Hopf subalgebra, of the Hopf algebra of all representative functions on $G$, which contains the coordinate ring of $G$.

Using parts (a) and (b) of (1.1), we have a rational action $G^{\prime} \times G \rightarrow G$. Following [6] we let $\phi: G^{\prime} \rightarrow G$ be given by $\phi(g)=g \cdot e$. If $H=\left\{g^{\prime} \in\right.$ 
$\left.G^{\prime} \mid \phi\left(g^{\prime}\right)=e\right\}$, then we have an isomorphism $G^{\prime} / H \rightarrow G$ (of varieties) given by $g^{\prime} H \rightarrow \phi\left(g^{\prime}\right)$.

Proposition 1.2. Let $G$ be a solvable analytic left algebraic group and let $G^{\prime}$ be as in (1.1). Let $N$ be a normal subgroup of $G$ such that $p(N)$ is a unipotent algebraic subgroup of $G^{\prime}$. Then the analytic group $G / N$ is an analytic left algebraic group such that $G \rightarrow G / N$ is a morphism.

Proof. Since $p(G)$ is dense in $G^{\prime}$ and normalizes $p(N)$, while $p(N)$, and hence its normalizer, are closed in $G^{\prime}, p(N)$ is normal in $G^{\prime}$. We identify $N$ with $p(N)$ via $p$. Let $H=\left\{g^{\prime} \in G^{\prime} \mid \phi\left(g^{\prime}\right)=e\right\}$. Then $N H$ is an algebraic subgroup of $G^{\prime}$ and it is straightforward to check that $G^{\prime} / N H \rightarrow G / N$ by $g^{\prime} N H \rightarrow N \phi\left(g^{\prime}\right)$ is a bijection such that the following diagram commutes:<smiles>CCCNC#N</smiles>

This gives the set $G / N$ an algebraic variety structure compatible with that of $G$. By (1.1(c)), $N$ acts rationally on $G$, and it is clear that $G / N$ is the quotient of $G$ under this action. Now $G^{\prime}$ and $N H$ are both solvable, so it follows from [2, Corollary, p. 5-02] that $G^{\prime} / N H$ and hence $G / N$ is an affine variety. Then $\mathbf{C}[G / N]=\mathbf{C}[G]^{N}$ consists of representative analytic functions on $G / N$, and since $\mathbf{C}[G]$ is right $G$-stable and $N$ is normal, $\mathbf{C}[G]^{N}$ is also right $G / N$-stable. Thus $\mathbf{C}[G / N]$ gives an analytic left algebraic group structure on $G / N$.

In the course of the proof we remarked on the variety structure of a solvable analytic left algebraic group. For future reference we record this in the following corollary:

COROLlary 1.3. Let $G$ be a simply connected solvable analytic left algebraic group. Then, as an algebraic variety, $G$ is isomorphic to $\mathbf{C}^{(n)}$ for some integer $n$.

Proof. In the notation of (1.3) we have an isomorphism $G^{\prime} / H \rightarrow G$ of algebraic varieties. Let $H_{0}$ be the connected component of the identity of $H$ and let $\Gamma$ be the finite group $H / H_{0}$. By [2, Corollary, p. 5-02], $G^{\prime} / H_{0}$ is an affine variety and $G^{\prime} / H_{0} \rightarrow G^{\prime} / H=G$ displays $G$ as the quotient of $G^{\prime} / H_{0}$ by $\Gamma$. But $\Gamma$ acts freely on $G^{\prime} / H_{0}$ so this is a covering space of the simply connected space $G$, and hence $\Gamma$ is trivial. Now by [2, Corollary, p. 5-02] again, $G^{\prime} / H=\mathbf{C}^{(n)} \times(\mathbf{C}-0)^{(m)}$ and since $G$ is simply connected, $m=0$.

We now begin the structure theory of simply connected solvable analytic left algebraic groups. We need the following definition:

Definition 1.4. (a) Let $G$ be an analytic group. A normal analytic subgroup $N$ of $G$ is called co-nilpotent if $G / N$ is nilpotent. The intersection of all the co-nilpotent subgroups of $G$ is called the minimal co-nilpotent subgroup of $G$. 
(b) Let $L$ be a finite dimensional Lie algebra. An ideal $I$ of $L$ is called co-nilpotent if $L / I$ is nilpotent. The intersection of all the co-nilpotent ideals of $L$ is the minimal co-nilpotent ideal of $L$.

For later use we record some obvious consequences of the definition.

ReMARKs 1.5. (a) If $G$ is an analytic group with minimal co-nilpotent subgroup $N, \operatorname{Lie}(N)$ is the minimal co-nilpotent ideal of $\operatorname{Lie}(G)$.

(b) If $G$ is a simply connected solvable analytic left algebraic group with minimal co-nilpotent subgroup $N$, then by (1.1(d)) and (1.2) the group $G / N$ is algebraic and $G \rightarrow G / N$ is a morphism of left algebraic groups with kernel $N$. Since any algebraic group which is a homomorphic image of $G$ must be unipotent, it follows that $N$ coincides with the algebraic kernel of $G$ defined in [6, Definition 1.12].

In [6, Theorem 3.1] it is shown how a simply connected solvable analytic group can be given a left algebraic group structure such that the group is generated by a normal nilpotent subgroup and another nilpotent subgroup. (See also [3, p. 114].) The following theorem shows that this is the case for all left algebraic structures on such a group.

THEOREM 1.6. Let $G$ be an analytic left algebraic group which is simply connected and solvable as an analytic group, and let $N$ be the minimal co-nilpotent subgroup of $G$. Then $N$ is a Zariski-closed unipotent normal subgroup of $G$, and there is a Zariski-closed unipotent subgroup $C$ of $G$ such that $N C=G$.

Proof. We argue by induction on the dimension of $G$. The case $\operatorname{dim} G=0$ being trivial. Since $N$ is an analytic subgroup of $(G, G)$ we know by (1.1(c)) that $N$ is a Zariski-closed unipotent subgroup of $G$. If $N_{0}$ is a proper analytic subgroup of $N$ which is normal in $G$, then by (1.1(d)) and (1.2), $\tilde{G}=G / N_{0}$ is an analytic left algebraic group and $G \rightarrow \tilde{G}$ is a morphism. It is clear that the minimal co-nilpotent subgroup of $\tilde{G}$ is $\tilde{N}=N / N_{0}$. By induction, there is a closed unipotent subgroup $\tilde{C}$ of $\tilde{G}$ with $\tilde{G}=\tilde{N} \tilde{C}$. We have $\tilde{C}=C^{\prime} / N_{0}$ for some closed left algebraic subgroup $C^{\prime}$ of $G$. Since $N_{0}$ is a proper subgroup of $N, \tilde{G}$ is not nilpotent so $C^{\prime}$ is a proper subgroup of $G$. By induction, $C^{\prime}=N^{\prime} C$, where $N^{\prime}$ is the minimal co-nilpotent of $C^{\prime}$ and $C$ is a closed unipotent subgroup of $C^{\prime}$ (and hence closed in $G$ ). Since $C^{\prime} / N_{0}=\tilde{C}$ is nilpotent, $N^{\prime}$ is contained in $N_{0}$, hence in $N$. Thus $G=N C$, as desired.

It remains to consider the case that $N$ has no proper normal analytic subgroup which is normal in $G$. Since if $N=\{e\}$ then $G=C$, we may assume $N \neq\{e\}$. Since $(N, N)$ is an analytic subgroup of $N$ normal in $G$, and since $N$ is nilpotent, we must have $(N, N)=\{e\}$. Let $g$ be the Lie algebra of $G$ and let $\Re$ be the Lie algebra of $N$. Then $[\Re, \Re]=0$, and hence, under the adjoint action, $g / \Re$ acts on $\Re$. By (1.5(a)) $g / \Re$ is nilpotent, and so 
there is a nonzero vector $x$ in $\Re$ which is a joint characteristic vector for $g$. The span of $x$ is then a nonzero ideal of $g$ contained in $\Re$, hence equal to $\Re$ under our assumptions. Thus $N$ is one dimensional.

Now, let $G^{\prime}$ be the algebraic hull of $G$ as in (1.1) and let $\phi: G^{\prime} \rightarrow G$ be the retraction. Let $\bar{G}=G / N$, and let $\bar{G}^{\prime}=G^{\prime} / N$ (as in (1.2), $N$ is normal in $G^{\prime}$ ). Since $G$ is dense in $G^{\prime}, \bar{G}$ is dense in $\bar{G}^{\prime}$ and hence $\bar{G}^{\prime}$ is a nilpotent algebraic group, so $\bar{G}^{\prime}=\bar{U} \times T$ where $\bar{U}$ is unipotent and $T$ is a torus. Consider the analytic homomorphism $\bar{G} \rightarrow \bar{G}^{\prime} \rightarrow \bar{U}$, and suppose it has kernel $K$. Since $\bar{G} \rightarrow \bar{G}^{\prime}$ is injective, the induced map $K \rightarrow T$ is injective.

Let $\bar{\phi}: \bar{G}^{\prime} \rightarrow \bar{G}$ be the map induced by $\phi$, let $\bar{H}=\left\{x \in \overline{G^{\prime}} \mid \bar{\phi}(x)=e\right\}$ and let $\bar{H}_{0}$ be the connected component of $e$ in $\bar{H}$. Then $\bar{H}_{0}=U_{1} \times T_{1}$ where $U_{1}$ is a unipotent subgroup of $\bar{U}$ and $T_{1}$ is subtorus of $T$. Then $\bar{G}^{\prime} / \bar{H}_{0}=\bar{U} / U_{1}$ $\times T / T_{1} \rightarrow \bar{G}^{\prime} / \bar{H}=\bar{G}$ is finite étale. It follows from (1.3) that $T_{1}=T$ and $\bar{U} / U_{1}$ is isomorphic to $\bar{G}$. But $\bar{G} \rightarrow \bar{U}$ is an injection with dense image of simply connected nilpotent groups, so an isomorphism, so $\operatorname{dim}(\bar{G})=\operatorname{dim}(\bar{U})$ and $U_{1}=\{e\}$. It follows that $\bar{H}=\bar{H}_{0}=T$. Now let $H=\left\{x \in G^{\prime} \mid \phi(x)=\right.$ $e\}$, and let $p: G^{\prime} \rightarrow \bar{G}^{\prime}$ be the projection. By the above, $p(H)<T$. Since $H \wedge N=\{e\}, p$ induces an injection $q: H \rightarrow T$. Since $\operatorname{dim}(H)=\operatorname{dim}\left(G^{\prime}\right)-$ $\operatorname{dim}(G)=\operatorname{dim}\left(\bar{G}^{\prime}\right)-\operatorname{dim}(\bar{G})=\operatorname{dim}(T), q$ is an isomorphism, and hence $H$ is a torus in $G^{\prime}$. It follows that there is a Cartan subgroup $C^{\prime}$ of $G^{\prime}$ containing $H$. Let $U$ be the unipotent radical of $C^{\prime}$. Then $H$ centralizes $U$. If $x$ is in $G^{\prime}$, then $\phi(x)$ is the unique element of $G$ such that $\phi(x) H=x H$. It follows that if $u_{1}, u_{2}$ are in $U$, then $\phi\left(u_{i}\right)=u_{i} h_{i}$ where $h_{i} \in H$. Then $\phi\left(u_{1}\right) \phi\left(u_{2}\right)=u_{1} h_{1} u_{2} h_{2}$ $=u_{1} u_{2} h_{1} h_{2}$ since $H$ centralizes $U$. Thus $\phi\left(u_{1}\right) \phi\left(u_{2}\right) H=u_{1} u_{2} H$ so $\phi\left(u_{1} u_{2}\right)=$ $\phi\left(u_{1}\right) \phi\left(u_{2}\right)$, and $\phi \mid U$ is a homomorphism. Let $C=\phi(U)$. We claim that $C$ is the desired subgroup of $G$. By [4, p. 81], $C^{\prime}$ maps onto $\bar{G}^{\prime}$, and since $G^{\prime}$ is not nilpotent, $C^{\prime} \neq G^{\prime}$. Since $N$ is one dimensional, $C^{\prime} \wedge N=\{e\}$ and $C^{\prime} \rightarrow \bar{G}^{\prime}$ is an isomorphism, so $U \rightarrow \bar{U}$ is an isomorphism. Also, $U \wedge H=\{e\}$, so $\phi \mid U$ is a bijection, so $C \rightarrow \bar{G}$ is a bijection. Now $C$ is the image of a morphism of varieties, hence constructible in $G$. Since $C=\phi(U)=U \phi(e)$ is homogeneous, $C$ is open in its closure and hence $C$ is a subvariety of $G$. By (1.1(c)), $h$ : $N \times C \rightarrow G$ is a morphism which is bijective by the above remarks. Since $N \times C$ and $G$ are nonsingular, and we are in characteristic zero, $h$ is an isomorphism. It follows that $C$ is closed in $G$, and this completes the proof of (1.6).

The following example may help illustrate the proof of (1.6): let $G=\mathbf{C}^{(2)}$ with multiplication $(a, b)(c, d)=\left(a+e^{b} c, b+d\right)$. As shown in [6], $G^{\prime}=$ $\mathbf{C}^{(2)} \times(\mathbf{C}-0)$ with multiplication $(x, y z)\left(x^{\prime}, y^{\prime}, t^{\prime}\right)=\left(x+z x^{\prime}, y+y^{\prime}, z z^{\prime}\right)$. The map $G \rightarrow G^{\prime}$ is $(a, b) \rightarrow\left(a, b, e^{b}\right)$ while $\phi: G^{\prime} \rightarrow G$ is $(x, y z) \rightarrow(x, y)$. The minimal co-nilpotent subgroup $N$ of $G$ is $\mathbf{C} \times\{0\}$, and the Cartan subgroup $C^{\prime}$ of $G^{\prime}$ complementing $N$ is $\{0\} \times \mathbf{C} \times(\mathbf{C}-0)$. Then $U=\{0\} \times$ 
$\mathbf{C} \times\{1\}$, and $\phi(U)=\{0\} \times \mathbf{C}=\mathbf{C}$, and it is clear that $G=N C$, with $N$ and $C$ possessing the properties of (1.6).

Also in [6, Corollary 3.5] it was shown that an analytic left algebraic group $G$ which is simply connected and solvable can be written as $N^{\prime} C^{\prime}$, where $N^{\prime}$ is the algebraic kernel of $G$ and $C^{\prime}$ is the core of $G$. By (1.5(b)), and the observation that $C^{\prime}$ is nilpotent, the result (1.6) follows. On the other hand, the proof of [6, Corollary 3.5] uses the fact that all left algebraic group structures on a simply connected solvable group are conjugate, established in $[3,4.1$, p. 120]. As was explained above, we are going to establish the conjugacy result here by different means, and indeed (1.6) will be used in that argument.

Suppose that $G, N$, and $C$ are as in (1.6). By (1.1(c), (d)), $N \times C \rightarrow G$ by multiplication is a morphism of varieties. Let $g=\operatorname{Lie}(G), \Re=\operatorname{Lie}(N)$ and $\mathrm{C}=\operatorname{Lie}(C)$. The algebraic variety structures on $N$ and $C$ are those of unipotent subgroups of an algebraic hull $G^{\prime}$ of $G$, so that exp: $\Re \rightarrow N$ and exp: $\mathcal{C} \rightarrow C$ are isomorphisms of varieties. By [6, §3], $C(G)$, the core of $G$, is $N_{0} \cdot C$, where $N_{0}=\left\{n \in N \mid c \rightarrow c n c^{-1}\right.$ is a morphism from $C$ to $\left.N\right\}$. Finally, $\varkappa$ is an ideal of $g$, and hence stable under the adjoint action of $C$. Let $\Sigma \varkappa_{\alpha}$ be the root-space decomposition of $\Re$ as a module over the nilpotent Lie algebra $\mathcal{C}$. In this notation, we have the following result.

Lemma 1.7. $N_{0}=\exp (\overbrace{0})$.

Proof. Let $n=\exp (y)$ be in $N$. Then $n$ is in $N_{0}$ if and only if

$$
\exp (x) \rightarrow \exp (x) \exp (y) \exp (x)^{-1}=\exp (\operatorname{Exp}(a d x)(y))
$$

is a morphism from $C$ to $N$, i.e., if $x \rightarrow \operatorname{Exp}(a d x)(y)$ is a polynomial map from $\mathcal{C}$ to $\Re$. Write $y=\Sigma y_{\alpha}$ for $y_{\alpha} \in \mathfrak{R}_{\alpha}$. Then, for sufficiently large $m$, we have $\operatorname{Exp}(a d(x)(y))=\Sigma \exp (\alpha(x)) z_{\alpha}$, where $z_{\alpha}=y_{\alpha}+\cdots+(a d x-$ $\alpha(x))^{m} y_{\alpha} / m$ !. Since $x \rightarrow z_{\alpha}$ is a polynomial map, the full map will be polynomial if and only if $z_{\alpha}=0$ for $\alpha \neq 0$ (for all $x$ ). For fixed $x$, however, the nonzero terms in the expression for $z_{\alpha}$ are linearly independent, so if $y_{\alpha} \neq 0, z_{\alpha} \neq 0$. It follows that $x \rightarrow \operatorname{Exp}(a d x)(y)$ is a polynomial map exactly when $y_{\alpha}=0$ for $\alpha \neq 0$, i.e., when $y \in \mathcal{N}_{0}$.

We continue to retain the above notation. It is clear that $\operatorname{Lie}(C(G))=\tau_{0}$ $+\mathcal{~}$. The next result shows that this is actually a Cartan subalgebra of $g$.

LEMMA 1.8. $\Re_{0}+\mathcal{C}$ is a Cartan subalgebra of $g$.

Proof. We first observe that $\Re_{0}+C$ is a nilpotent subalgebra of $g$, since it is generated by the subset $\tau_{0} \cup \mathcal{C}$ which is closed under bracket, and each element of $\pi_{0} \cup \mathcal{C}$ is ad-nilpotent on $\pi_{0}+\mathcal{C}$. Now let $x$ be in $g$ such that $\Re_{0}+\mathcal{C}$ is nilpotent on $x$. Write $x=x_{1}+y$ with $y$ in $\Re$ and $x_{1}$ in $C$. Since $\mathcal{C}$ is nilpotent on $x$ and $x_{1}, \mathcal{C}$ is nilpotent on $y$, so $y$ is in $\Re_{0}$, and hence $x$ is 
in $\varkappa_{0}+\mathcal{C}$. It follows that $\varkappa_{0}+\mathcal{C}$ is a Cartan subalgebra of $g$.

It is useful to observe here that, even though $\Re$ is a minimal co-nilpotent ideal and $g=\Re+\mathcal{C}, \mathcal{C}$ need not be a Cartan subalgebra of $g$ : for consider the Lie algebra $L$ with basis $x, y, t, s$ and multiplication $x y=0, t y=x$, $t x=0, s x=0, s y=-y, s t=t$. Then the minimal co-nilpotent ideal is $[L$, $L]$ with basis $x, y, t$ and $L$ can be written as $[L, L]+\mathrm{Cs}$, but $\mathrm{Cs}$ is not a Cartan subalgebra of $L$, since $x$ is in its normalizer. (This example is the Lie algebra of the example in $[6, \S 3]$.)

In [6, Definition 2.2] the algebraic Lie algebra $L(G)$ of an analytic left algebraic group $G$ was defined as the set of all $G$-invariant derivations of $\mathrm{C}[G]$, and in [6, Theorem 2.5] it was shown that $L(G)=\operatorname{Lie}(C(G))$. Combining (1.6), (1.7) and (1.8), we obtain the following characterization of $L(G)$ :

COROLLARY 1.9. Let $G$ be an analytic left algebraic group, simply connected and solvable as an analytic group. Then $L(G)$ is a Cartan subalgebra of $\operatorname{Lie}(G)$.

Now Cartan subalgebras of a given Lie algebra are conjugate, and, using (1.9), it follows that the cores corresponding to different left algebraic group structures on a given simply connected solvable analytic group are conjugate. We will use this below to show that the left algebraic group structures are actually conjugate. First, we show that the core determines the left algebraic group structure.

THEOREM 1.10. Let $G$ be a simply connected solvable analytic group and let $\mathcal{C}$ be a Cartan subalgebra of $\operatorname{Lie}(G)$. Then there exists a unique left algebraic group structure on $G$ such that $L(G)=\mathcal{C}$.

Proof. Let $\Re$ be the minimal co-nilpotent ideal of $\operatorname{Lie}(G)$. Then $\Re$ is a nilpotent Lie algebra and $\Re+\mathcal{C}=\operatorname{Lie}(G)$. Let $N=\exp (\Re)$ and $C=$ $\exp (\mathcal{C})$. By [3, pp. 114-116] we can find a left algebraic group structure on $G$ such that $N \times C \rightarrow G$ is a morphism, where $N \times C$ is regarded as a semidirect product left algebraic group, and such that $C$ is contained in $C(G)$. But then $\mathcal{C}=\operatorname{Lie}(C)$ is contained in $L(G)$, and by (1.9) $\mathcal{C}=L(G)$. This establishes the existence assertion of (1.10). For uniqueness, we begin by observing that $N$ is the minimal co-nilpotent subgroup of $G$. Suppose we have some left algebraic group structure on $G$ with core $C^{\prime}$. By (1.6), and the remarks preceding (1.7) the semidirect product left algebraic group $N \times C^{\prime}$ maps to $G$ morphically. If $\operatorname{Lie}\left(C^{\prime}\right)=\mathcal{C}$, then $C^{\prime}=C$ so that $N \times C^{\prime}=N \times$ $C$, and hence the left algebraic group structure on $G$ is that constructed in the first part of the proof, which is therefore unique.

Now we can establish the conjugacy result:

THEOREM 1.11. Let $G$ be a simply connected solvable analytic group and let $N$ 
be the minimal co-nilpotent subgroup of $G$. Suppose that $(G, A)$ and $\left(G, A^{\prime}\right)$ are analytic left algebraic group structures on $G$. Then there exists an $x$ in $N$ such that $x \cdot A=B$.

Proof. Let $C$ and $C^{\prime}$ denote the cores associated to $A$ and $A^{\prime}$, respectively, and let $C=\operatorname{Lie}(C)$ and $\mathcal{C}^{\prime}=\operatorname{Lie}\left(C^{\prime}\right)$. By (1.10), we need to find $x$ in $N$ such that $x C x^{-1}=C^{\prime}$. It will suffice to find $y$ in $\operatorname{Lie}(N)$ such that $\operatorname{Exp}(a d y)(e)=$ $\mathcal{C}^{\prime}$. The element $y$ of $\operatorname{Lie}(G)$ is strongly ad-nilpotent $[4$, p. 82] if there is $z$ in $\operatorname{Lie}(G)$ such that $(a d z-a)^{n}(y)=0$ for some $a \neq 0$. By [4, Theorem 16.2, p. 82] there is a sequence $y_{1}, \ldots, y_{n}$ of strongly ad-nilpotent elements of $\operatorname{Lie}(G)$ such that $\operatorname{Exp}\left(a d y_{1}\right) \circ \cdots \circ \operatorname{Exp}\left(a d y_{n}\right)(C)=\mathcal{C}^{\prime}$. To complete the proof, we observe that each strongly ad-nilpotent element of $\operatorname{Lie}(G)$ must belong to the minimal co-nilpotent ideal, and in this case that ideal is $\operatorname{Lie}(N)$.

As a consequence of (1.10), we can also determine automorphism groups.

Proposition 1.12. Let $G$ be an analytic left algebraic group, simply connected and solvable as an analytic group. Then the automorphism group of $G$ as a left algebraic group is isomorphic to the group of automorphisms of $\operatorname{Lie}(G)$ which carry $L(G)$ to $L(G)$.

Proof. The automorphism group of $G$ as an analytic group is isomorphic to the automorphism group of $\operatorname{Lie}(G)$, and an automorphism of $G$ as a left algebraic group preserves $C(G)$, so the corresponding automorphism preserves $L(G)$. Conversely, an automorphism $s$ of $\operatorname{Lie}(G)$ preserving $L(G)$ comes from an analytic automorphism $t$ of $G$ preserving $C(G)$, and then $t^{*} \mathbf{C}[G]$ is a left algebraic group structure on $G$ with core $C(G)$. By (1.10), $t^{*} \mathbf{C}[G]=\mathbf{C}[G]$, and hence $t$ is a left algebraic group automorphism of $G$.

We will use $(1.12)$ and $[5,3.1$, p. 267] to construct semidirect products of left algebraic groups below.

2. We recall [6, Definition 3.6] that an analytic left algebraic group $G$ is called normal basic if $G$ is a semidirect product as left algebraic group of a normal, simply connected solvable subgroup $K$ and a reductive subgroup $H$. Then $H$ is a maximal reductive subgroup of $G$ and $K$ is a nucleus of $G$, in the sense of [3, p. 112]. By [6, 3.7], $C(G)=C(K) H$.

Since conjugation by $H$ acts as automorphisms of the left algebraic group $K, C(K)$ must be stable under conjugation by $H$. Using the adjoint representation, we have [Lie $H, \operatorname{Lie}(C(K))] \subset \operatorname{Lie}(C(K))$. By $(1.10), \operatorname{Lie}(C(K))$ is a Cartan subalgebra of $\operatorname{Lie}(K)$, stable under $\operatorname{Lie}(H)$. Conversely, suppose $G=K_{1} H_{1}$ is any decomposition of $G$ as an analytic semidirect product of a simply connected solvable normal subgroup $K$, and a reductive subgroup $H_{1}$. We claim that there is a Cartan subalgebra $C$ of $\operatorname{Lie}\left(K_{1}\right)$ stable under $\operatorname{Lie}\left(H_{1}\right)$. By $\left[3,2.1\right.$, p. 113] we can write $\operatorname{Lie}\left(K_{1}\right)=S_{1}+S_{2}$ where $S_{1}$ is a nilpotent 
ideal of $\operatorname{Lie}\left(K_{1}\right)$ stabilized by $\operatorname{Lie}\left(H_{1}\right)$ and $S_{2}$ is a nilpotent subalgebra of $\operatorname{Lie}\left(K_{1}\right)$ commuting with $\operatorname{Lie}\left(H_{1}\right)$. Let $S^{\prime}$ be the 0 root space of $S_{2}$ on $S_{1}$. Then, as in (1.8), $S^{\prime}+S_{1}$ is a Cartan subalgebra of $\operatorname{Lie}\left(K_{1}\right)$, and since $\left[\operatorname{Lie}\left(H_{1}\right), S_{1}\right]=0, S^{\prime}$, and hence also $S^{\prime}+S_{1}$, is $\operatorname{Lie}\left(H_{1}\right)$-stable. Give $K_{1}$ the analytic left algebraic group structure with $\operatorname{Lie}\left(C\left(K_{1}\right)\right)=\mathcal{C}$. By (1.12), $H_{1}$ acts, via conjugation, as left algebraic group automorphisms of $K_{1}$, so, under the canonical algebraic group structure on $H_{1}, G=K_{1} H_{1}$ is actually a semidirect product of left algebraic groups.

In $[3,3.1, \mathrm{p} .118]$, it is shown that a normal basic left algebraic group structure determines a nucleus. We will show that the core of such a structure determines the nucleus.

LEMMA 2.1. Let $G=K \cdot H$ be a normal basic analytic left algebraic group, let $\mathcal{C}$ be the Lie algebra of the unipotent radical of $C(G)$, and let $\Re$ be the ideal of $\operatorname{Lie}(G)$ generated by the root spaces of the nonzero roots of $\mathcal{C}$ on $\operatorname{Lie}(G)$. Then $\operatorname{Lie}(K)=\Re+C$.

Proof. We know that $\operatorname{Lie}(G)=\operatorname{Lie}(K)+\operatorname{Lie}(H)$, as a semidirect product, and that $\mathcal{C}=\operatorname{Lie}(C(K))$, since $C(G)=C(K) \cdot H$, and that $[\operatorname{Lie}(H), \mathcal{C}]$ is contained in $\mathcal{C}$. By (1.9), $\mathcal{C}$ is a Cartan subalgebra of $\operatorname{Lie}(K)$, and hence the root space decomposition of $\operatorname{Lie}(K)$ under $G$ is $\Sigma_{\alpha \neq 0}(\operatorname{Lie}(K))_{\alpha}+\mathcal{C}$. Since $[\operatorname{Lie}(H), \mathcal{C}] \subset \mathcal{C}$, the zero root space of $\operatorname{Lie}(G)$ is $\mathcal{C}+\operatorname{Lie}(H)$. It follows that the $\operatorname{Lie}(K)_{\alpha}$, for $\alpha \neq 0$, are the root spaces for the nonzero roots of $\mathcal{C}$ on $\operatorname{Lie}(G)$, and hence $\Re$ is contained in $\operatorname{Lie}(K)$, and it is clear that $\Re+e=$ $\operatorname{Lie}(K)$.

We will also use the following elementary remark:

LEMMA 2.2. Let $G=K H$ be a normal basic analytic left algebraic group and let $H_{1}$ be a maximal reductive subgroup of $C(G)$. Then $G$ is a semidirect product $G=K H_{1}$ as left algebraic groups.

Proof. There is an element $g$ of $C(G)$ such that $g \mathrm{Hg}^{-1}=H_{1}$. Since $g$ is in $C(G)$, inner automorphism by $g$ is an automorphism of $G$ as left algebraic group, which carries $K$ to $K$ and $H$ to $H_{1}$, so the result follows.

Combining Lemmas (2.1) and (2.2), we see that the core of a normal basic $G=K H$ essentially determines both $H$ and $K$. In fact, we can now show that the core determines the left algebraic group structure.

THEOREM 2.3. Let $G$ be an analytic group and suppose $(G, A)$ and $\left(G, A^{\prime}\right)$ are normal basic analytic left algebraic group structures on $G$ with corresponding cores $C$ and $C^{\prime}$. If $C=C^{\prime}$ (as algebraic groups), then $A=A^{\prime}$.

Proof. Let $G=K H=K^{\prime} H^{\prime}$ be the corresponding semidirect product decompositions associated to the structures $A$ and $A^{\prime}$. Then $C=C(K) H=$ $C\left(K^{\prime}\right) H^{\prime}$ by hypothesis. Thus $H^{\prime}$ is a maximal reductive subgroup of $C$, so 
by (2.2) we can assume $H=H^{\prime}$. The unipotent radical of $C$ is $C(K)$ and that of $C^{\prime}$ is $C\left(K^{\prime}\right)$, so since $C=C^{\prime}$ as algebraic groups, $C(K)=C\left(K^{\prime}\right)$. By (2.1), $K=K^{\prime}$. By (1.10), there is a unique left algebraic group structure on $K$ with core $C(K)$, and thus $C$ determines $K$ as a left algebraic group. The result now follows.

The preceding theorem shows that normal basic left algebraic group structures on a given analytic group $G$ are determined by their cores. It is natural to inquire what properties an analytic subgroup of $G$ must have to be the core of some such structure. We know that such a core must be of the form $C \cdot H$, where $C$ is the core of some left algebraic group structure on a nucleus $K$ of $G$ and $H$ is a maximal reductive subgroup of $G$ normalizing $C$. Let $\mathcal{C}$ denote $\operatorname{Lie}(C), \mathcal{K}$ denote $\operatorname{Lie}(K), \mathcal{K}$ denote $\operatorname{Lie}(H)$, and $\mathcal{G}$ denote $\operatorname{Lie}(G)$. We are going to analyze the position of $\mathcal{C}$ inside of $\mathcal{G}$. First, we have that $[\mathcal{H}, \mathcal{C}$ ] is contained in $\mathcal{H}$. Let $\Re=\operatorname{rad}(\mathcal{G})$. It is clear that $\mathcal{K} \subset \Re$ and that $\Re=\mathscr{K}+\operatorname{rad}(\mathcal{C})$, and the latter sum is a semidirect product decomposition. Let $\Re=\Sigma \Re_{\alpha}$ be the root space decomposition of $\Re$ as a $C$-module. Then $\Re_{0}=\mathcal{C}+\operatorname{rad}(\mathcal{H})$, and, for $\alpha \neq 0, \Re_{\alpha}=\left[\mathcal{C}, \Re_{\alpha}\right]$ is contained in $[\Re$, $\Re]$ which is contained in $\mathscr{K}$ since $\operatorname{rad}(\mathcal{H})$ is abelian. In particular, if $\alpha \neq 0$, $\left[\Re_{\alpha}, \Re_{-\alpha}\right.$ ] is contained in $\mathcal{K} \cap \Re_{0}$ which equals $\mathcal{C}$. We now show that the conditions on $C$ we have determined are sufficient to guarantee that $C$ is the unipotent radical of a core of $G$.

THEOREM 2.4. Let $G$ be a faithfully representable analytic group and let $H$ be a maximal reductive subgroup of $G$. Let $\Re$ denote the radical of $\operatorname{Lie}(G)$, let $\mathcal{C}$ be a nilpotent subalgebra of $\Re$, let $\Sigma \Re_{\alpha}$ be the root space decomposition of $\Re$ as $a \mathcal{C}$-module and let $C=\exp (\mathcal{C})$. Then there is a normal basic left algebraic group structure on $G$ with $C(G)=C \cdot H$ if and only if the following conditions are satisfied:

(a) $\mathcal{C} \cap \operatorname{Lie}(H)=0$.

(b) $[\operatorname{Lie}(H), \mathcal{C}] \subset \mathcal{C}$.

(c) $\Re_{0}=e+\operatorname{rad}(\operatorname{Lie}(H))$.

(d) If $\alpha \neq 0$, [ $\left.\Re_{\alpha}, \Re_{-\alpha}\right] \subset \mathcal{C}$.

Proof. We have already seen that the conditions are necessary. We will show they are also sufficient. Let $\mathcal{S}=\mathcal{C}+\Sigma_{\alpha \neq 0} \Re_{\alpha}$. By (d), $\delta$ is a subalgebra of $\Re$, and by (b) $\mathcal{S}$ is even an ideal of $\operatorname{Lie}(G)$. By (c), $\Re=\mathcal{S}+$ $\operatorname{rad}(\operatorname{Lie}(H))$, and hence $\operatorname{Lie}(G)=\mathcal{S}+\operatorname{Lie}(H)$, and it is clear that this sum is direct. It is also clear that $\mathcal{C}$ is a Cartan subalgebra of $\mathcal{S}$. Since $\mathcal{S}$ is a subalgebra of $\Re, \delta$ is solvable. We want to show that $\delta$ is the Lie algebra of some nucleus of $G$. If $\alpha \neq 0,\left[\mathcal{C}, \Re_{\alpha}\right]=\Re_{\alpha}$, and this observation, plus condition (d) shows that $[\Re, \Re]$ is contained in $\mathcal{S}$ and that $\mathcal{S}=[\Re$, $\Re]+\mathcal{C}$. Now, by assumption, $G$ has a nucleus $K$, and since $[\Re, \Re] \subset$ $\operatorname{Lie}(K)$, we must have that $N=\exp ([\Re, \Re])$ is a simply connected nilpotent 
subgroup of $K$. Since $R$ is an ideal of $\operatorname{Lie}(G), N$ is normal in $G$. Now $C=\exp (\mathcal{C})$ is nilpotent subgroup of $G$, and hence $C=U \times T$ where $U$ is simply connected and $T$ is a complex toroid. Now $C$ is $H$-stable, and since $T$ is the unique maximal reductive subgroup of $C, H$ normalizes $T$. But then $T \cdot H$ is a reductive subgroup of $G$ properly containing $H$, contrary to the maximality of $H$, unless $T$ is trivial. Thus $C$ is simply connected. It follows that $S=N \cdot C$ is simply connected. Now $\delta=\operatorname{Lie}(S)$, and $S \cap H=\{e\}$, so $S$ is the desired nucleus with Lie algebra $S$. We give $S$ the left algebraic group structure with core $C$, which exists since $\mathcal{C}$ is Cartan in $\mathcal{S}$. Now $H$ normalizes $C$, so we can give $G$ the semidirect product left algebraic group structure $G=S \cdot H$, and in this structure $C(G)=C(S) \cdot H=C \cdot H$, as desired.

Condition (c) of (2.4) implies that if $\alpha \neq 0,\left[\Re_{\alpha}, \Re_{-\alpha}\right] \subset \mathcal{C}+\operatorname{rad}(H)$. That the stronger condition (d) is actually required is shown by the following example: let $\boldsymbol{G}=\mathbf{C}^{(3)} \times(\mathbf{C}-\{0\})$ with multiplication

$$
(x, y, t, x)\left(x^{\prime}, y^{\prime}, t^{\prime}, s^{\prime}\right)=\left(x+x^{\prime}+x t y^{\prime}, y+s^{-1} t y^{\prime}, t+s t^{\prime}, s s^{\prime}\right) .
$$

$\operatorname{Lie}(G)$ is a four-dimensional algebra with basis $A, B, C, Y$ such that

$$
\exp (t A+y B+x C+s Y)=\left(x, y, t, e^{s}\right)
$$

and multiplication $A C=B C=Y C=0, A B=C, Y A=A, Y B=-B$. Clearly $H=0 \times(C-\{0\})$ is a maximal reductive subgroup of $G$ and $\operatorname{Lie}(H)=\mathbf{C} Y$. Let $Z=C-Y$ and let $\mathcal{C}=\mathbf{C} Z$. We have $Z Y=0, Z C=0$, $Z B=B, Z A=-A$. Then $C$ satisfies conditions (a), (b), (c) of (2.4) relative to $\operatorname{Lie}(H)$, but $A$ and $B$ are in nonzero root spaces of $C$ with $A B=C$ not in e.

It is known that left algebraic group structures which give rise to the same nucleus are equivalent under conjugation by the radical of the commutator subgroup of the group $[3,4.1$, p. 120]. We will derive a slightly stronger version of this result as a consequence of (1.11) and the following lemma.

LEMMA 2.5. Let $G$ be a normal basic left algebraic group, $C$ the core of $G$ and $U$ the unipotent radical of $G$. Then:

(a) the normalizer in $G$ of $U$ is $C$,

(b) the normalizer in $G$ of $C$ is $C$.

Proof. Let $K$ be the nucleus of $G$ corresponding to $C(2.1)$ and let $H$ be a maximal reductive subgroup of $G$ contained in $C$. Then $G=K \cdot H$, and $H$ normalizes $U$. Also $U$ is the core of $K$, so by (1.9) $U$ is a Cartan subgroup of $K$ and hence self-normalizing. Now if $g=k h$ with $k \in K$ and $h \in H$, then $g U g^{-1}=k U k^{-1}=U$ if and only if $k \in U$, hence the normalizer of $U$ is $U \cdot H=C$, and (a) is established. For (b), we note that $U H \cap K=U$, so if $g U \mathrm{Hg}^{-1}=U H, g$ normalizes $U$ and hence belongs to $C$.

THEOREM 2.6. Let $G$ be an analytic group, and let $(G, A)$ and $\left(G, A^{\prime}\right)$ be 
normal basic left algebraic group structures on $G$ corresponding to the same nucleus $K$. Let $N$ be the minimal co-nilpotent subgroup of $K$. Then there exists $x$ in $N$ such that $x \cdot A=A^{\prime}$.

Proof. By (1.11), after suitable conjugation by an element of $N$ we can assume that the induced structures on $K$ are equal. This means that if $C$ and $C^{\prime}$ are the cores of the $A$ and $A^{\prime}$ structures on $G$, then $C \cap K=C^{\prime} \cap K$ is the unipotent radical of $C$ and $C^{\prime}$. Now (2.5(a)) gives the result.

By (2.6) we know that cores coming from the same nucleus are conjugate. It is known [3, p. 121] that it is possible, in general, to have nonisomorphic nuclei, and so, by (2.1), nonconjugate left algebraic group structures are possible.

\section{REFERENCES}

1. A. Borel, Linear algebraic groups, Benjamin, New York, 1969. MR 40 \#4273.

2. A. Grothendieck, La torsion homologique et les sections rationnelles, Séminaire $\mathrm{C}$. Chevalley, 2e annee: 1958. Anneaux de Chow et Applications, Exposé 5, Secrétariat mathématique, Paris, 1958. MR 22 \# 1572.

3. G. Hochschild and G. D. Mostow, On the algebra of representative functions of an analytic group, Amer. J. Math. 83 (1961), 111-136. MR 25 \#5129.

4. J. Humphreys, Introduction to Lie algebras and representation theory, Springer-Verlag, Berlin and New York, 1972. MR 48 \#2197.

5. A. Magid, Left algebraic groups, J. Algebra 35 (1975), 253-272.

6. __ Analytic left algebraic groups, Amer. J. Math. (to appear).

7. M. Rosenlicht, On quotient varieties and the affine embedding of certain homogeneous spaces, Trans. Amer. Math. Soc. 101 (1961), 211-223. MR 24 \#1732.

Department of Mathematics, University of OkLahoma, Norman, OkLahoma 73069 